\title{
Interferometric Mapping of Magnetic Fields: The massive star forming region G34.4+0.23 MM
}

\author{
P. C. Cortes \\ Departamento de Astronomía, Universidad de Chile, Casilla 36-D Santiago, Chile \\ R. M. Crutcher \\ Astronomy Department, University of Illinois at Urbana-Champaign, IL 61801, USA \\ D. S. Shepherd ${ }^{1}$ \\ National Radio Astronomy Observatory, P.O. Box O, 1003 Lopezville Rd, Socorro, NM \\ 87801. \\ L. Bronfman \\ Departamento de Astronomía, Universidad de Chile, Casilla 36-D Santiago, Chile
}

\begin{abstract}
We report millimeter interferometric observations of polarized continuum and line emission from the massive star forming region G34.4. Polarized thermal dust emission at $3 \mathrm{~mm}$ wavelength and $\mathrm{CO} J=1 \rightarrow 0$ line emission were observed using the Berkeley-Illinois-Maryland Association (BIMA) array. Our results show a remarkably uniform polarization pattern in both dust and in CO $\mathrm{J}=1 \rightarrow 0$ emission. In addition, the line emission presents a consistent uniform polarization pattern over most of the velocity channel maps. These uniform polarization patterns are aligned with the north-south main axis of the filament between the main millimeter source (MM) and the ultra-compact $\mathrm{H}$ II region, which are the central sources in G34.4, suggesting a magnetic field orthogonal to this axis. This morphology is consistent with a magnetically supported disk seen roughly edge-on.
\end{abstract}

Subject headings: ISM: magnetic fields ISM:polarization stars: formation

\footnotetext{
${ }^{1}$ The National Radio Astronomy Observatory is a facility of the National Science Foundation operated under cooperative agreement by Associated Universities, Inc.
} 


\section{Introduction}

It is generally accepted that magnetic fields play an important role in the process of star formation; magnetic fields are involved in cloud support, fragmentation, and transfer of angular momentum. However, the magnetic field is the least observed physical quantity involved in such process. Magnetic field observations of molecular clouds are divided into measurements of the line-of-sight component of the magnetic field strength through the Zeeman effect and observations of the field in the plane of the sky through linear polarization of dust emission and spectral-line emission. The alignment of dust grains by a magnetic field is physically complicated and is still a matter of intense research. It is accepted, though, that aligned dust grains will produce polarized emission perpendicular to the projection of the magnetic field onto the plane of the sky. For a recent review of alignment theories see Lazarian (2007).

Spectral-line linear polarization has been suggested to arise from molecular clouds under anisotropic conditions, like large velocity gradients (or LVGs) (Goldreich \& Kylafis 1981). The prediction suggests that a few percent of linearly polarized radiation should be detected from molecular clouds and circumstellar envelopes in the presence of a magnetic field. This polarization will be either parallel or perpendicular to the projection of the field onto the plane of the sky. To obtain a qualitative understanding about this effect, consider the CO molecule emitting unpolarized radiation. Under a magnetic field, a CO molecule will develop a small splitting in its rotational, $J$, energy levels. These magnetic sub-levels will produce radiation components labeled $\sigma$ for the $|M-M|=1$ transitions, and $\pi$ for the $|M-M|$ $=0$ transitions, where both components can be linearly polarized either perpendicular or parallel to the magnetic field. If the gas behaves under isotropic conditions (e.g. no velocity gradients) for any direction the $\sigma$ and $\pi$ will populate equally, so the radiation components emerging from the radiative decays of these states will combine to give zero net polarization. Now on the other hand, large velocity gradients present in molecular clouds will produce anisotropies in the optical depths for the CO molecular transitions at different directions. If the velocity gradients are smaller in directions parallel to the magnetic field than in those perpendicular to the field, the optical depths parallel to the field will be larger than the optical depths perpendicular to them. Therefore, the escape of radiation involved in deexciting the upper $J$ state will be then reduced more in directions along the field lines than in directions perpendicular to them, which will lead to populations of the magnetic $\sigma$ sub-states that are larger than the populations of the $\pi$ sub-states due to the difference in the angular distributions of both radiation components. The angular distribution of the $\sigma$ component peaks in directions along the field lines whereas the $\pi$ component peaks in directions perpendicular to the magnetic field. In this way, the rate of de-excitation for

$\sigma$ will have a larger decrease, due to photon trapping, than the rate of de-excitation for 
the $\pi$ radiation component. Because, in this picture, the $\sigma$ component will have a larger population, its emission will be stronger relative to the $\pi$ component giving raise to a small amount of linear polarization in the $\mathrm{CO}$ emission with the polarization of the $\sigma$ component, or perpendicular to the magnetic field. The effect was first detected by Glenn et al. (1997) for the CS molecules while Greaves et al. (1999) detected CO polarized emission for $(J=2 \rightarrow 1)$ and $(J=3 \rightarrow 2)$ transitions.

In order to efficiently map polarized emission and infer detailed information about the magnetic field morphology, high resolution observations are required. The BIMA millimeter interferometer has been used previously to obtain high-resolution polarization maps in several star forming cores (Lai 2001; Lai et al. 2002, 2003; Cortes et al. 2005; Cortes \& Crutcher 2006; Cortes et al. 2006). These previous results show fairly uniform polarization morphologies over the main continuum sources, suggesting that magnetic fields are strong, and therefore cannot be ignored by star formation theory. However, the number of star formation regions with maps of magnetic fields remains small, and every new result is statistically significant. In this work we present polarization maps of the massive star forming region G34.4, obtained with the BIMA array. We measured continuum polarization at $3 \mathrm{~mm}$ and $\mathrm{CO} J=1 \rightarrow 0$ line polarization, obtaining interferometric maps for both line and continuum. The remainder of this paper is divided into five sections. Section 2 reviews information about the source, section 3 describes the observation procedure, section 4 presents the results, section 5 gives the discussion, and section 6 the conclusions and summary.

\section{Source Description}

G34.4 is a newly discovered massive star forming region. It is associated with the IRAS $18507+0121$ point source, which is located at $3.9 \mathrm{kpc}$ from the Sun, having a $v_{\mathrm{lsr}}=57 \mathrm{~km}$ $\mathrm{s}^{-1}$. Towards the IRAS $18507+0121$ point source, Bronfman et al. (1996) detected strong $\mathrm{CS}(J=2 \rightarrow 1)$ emission with broad line wings that suggested massive star formation. The source is roughly $11^{\prime}$ from the H II region complex G34.3+0.2 (Molinari et al. 1996). Observations with the $45-\mathrm{m}$ Nobeyama radio telescope at a resolution of $16^{\prime \prime}$ of $\mathrm{HCO}^{+}$, $\mathrm{H}^{13} \mathrm{CO}^{+}$, CS, and $\mathrm{C}^{34} \mathrm{~S}$ were presented by Ramesh et al. (1997). Their modeling showed that the observed line profiles are representative of a collapsing warm $(22 \mathrm{~K})$ core with a mass of $800 M_{\odot}$, hidden behind $\mathrm{a} \approx 4 \mathrm{~K}$ cold screen of about $200 M_{\odot}$. This region is also associated

with $\mathrm{H}_{2} \mathrm{O}$ maser emission (Scalise et al. 1989; Palla et al. 1991; Miralles et al. 1994) and $\mathrm{CH}_{3} \mathrm{OH}$ maser emission (Schutte et al. 1993; Szymczak \& Kus 2000). Faúndez et al. (2004) observed this region in the continuum at $1.2 \mathrm{~mm}$ with the SEST telescope and derived a mass of $2 \times 10^{3} \mathrm{M}_{\odot}$. Shepherd et al. (2004) performed the first interferometric observations with 
OVRO of the 3 -mm continuum, $\mathrm{H}^{13} \mathrm{CO}^{+}(J=1 \rightarrow 0)$, and $\mathrm{SiO}(v=0, J=2 \rightarrow 1)$. They detected two compact molecular cores along a north-south filament, separated by $\sim 40^{\prime \prime}$ (see Figure 1). They also presented near-infrared observations at J, H, and $\mathrm{K}^{\prime}\left(\lambda_{c}=1.25\right.$, 1.65, and $2.1 \mu \mathrm{m}$, respectively). The central source in Figure 2 (labeled G34.4+0.23 MM) showed no trace of NIR emission, but the southern source seemed to be associated with a NIR cluster of young stars and an ultra-compact $\mathrm{H}$ II region. Based on the emission from warm dust and the lack of NIR emission, the central source was suggested to be a massive proto-star. From the VLA archive, marginal emission $(0.7 \mathrm{mJy})$ of 6 -cm radio continuum was detected from this source (Shepherd et al. 2004). Rathborne et al. (2005) made a multiwavelength study of this region. They observed continuum emission at $1.2 \mathrm{~mm}, 850 \mu \mathrm{m}$, $450 \mu \mathrm{m}$, and $350 \mu \mathrm{m}$ by using IRAM, JCMT, and the CSO observatories respectively. They also obtained archival data from the SPITZER telescope at $2.4 \mu \mathrm{m}, 8 \mu \mathrm{m}, 4.5 \mu \mathrm{m}$, and $3.6 \mu \mathrm{m}$ and produced combined maps of infrared continuum emission. Their data agree well with previous observations and positioned the infrared sources at the center of the $\mathrm{mm}$ and submillimeter emission. In a recent study, Shepherd et al. (2007) presented a detailed study of the G34.4 region. They discovered five massive outflows from two of the existing cloud cores in G34.4. Three outflows are centered near the ultra-compact H II region while the remaining two are centered at the MM core. By using mid-IR data from the Spitzer

telescope, Shepherd et al. (2007) identified a total of 31 YSO in the G34.4 complex with a combined mass of $\sim 127 \mathrm{M}_{\odot}$ plus an additional 22 sources that might be cluster members based on strong $24 \mu \mathrm{m}$ emission.

\section{Observation Procedure}

We observed G34.4+0.23 MM in May 2004 in the 3-mm continuum and the CO $J=1 \rightarrow$ 0 molecular line (at $115 \mathrm{GHz}$ ); one track with the BIMA array in D configuration (at a resolution of $\left.16^{\prime \prime}\right)$ was obtained. The digital correlator was set up to observe both the continuum and the $\mathrm{CO} J=1 \rightarrow 0$ line simultaneously. The $750 \mathrm{MHz}$ wide lower side band was combined with $700 \mathrm{MHz}$ from the upper side band to map the continuum emission, leaving a $50 \mathrm{MHz}$ window for the CO line observation (at a resolution of $2.06 \mathrm{~km} \mathrm{~s}^{-1}$ ). The $50 \mathrm{MHz}$ window for $\mathrm{CO}$ was cut from the continuum window to avoid contamination of the continuum by the $\mathrm{CO}$ line and reduced independently. In order to detect circular polarization, a quarter-wave plate was placed in front of the single receiver at each BIMA antenna to select either right (R) or left (L) circular polarization. A second quarter-wave plate grooved orthogonally to the first was alternately switched into the signal path to observe sequentially both circular polarizations. Switching between polarizations was sufficiently rapid (every 11.5 seconds) to give essentially identical uv-coverage. Cross-correlating the $\mathrm{R}$ and $\mathrm{L}$ circularly polarized 
signals from the sky gave RR, LL, LR, and RL correlations for each interferometer baseline, from which maps of the four Stokes parameters were produced. The quasars 1751+096 and 1743-038 were used as calibrators for G34.4. The instrumental polarization was calibrated by observing the 3C279 quasar, and the "leakages" solutions were calculated from this ob-

servation. The calibration procedure is described by Lai (2001). The Stokes images I, U, $\mathrm{Q}$ and $\mathrm{V}$ were obtained by Fourier transforming the visibility data using a robust weighting scheme (Briggs et al. 1999). Deconvolution in the Stokes I cube was done by applying a maximum entropy algorithm to every channel. The MIRIAD (R. J. Sault, N. E. B. Killeen 1998) package was used for data reduction.

\section{Observational Results}

\subsection{3-mm Continuum}

The 3-mm continuum results are shown in Figure 2. The beam has a major axis of $17.6^{\prime \prime}$ and a minor axis of $15.7^{\prime \prime}$. The significance level cutoff chosen for the polarization results is $3 \sigma$, where $\sigma$ refers the noise level in the polarized flux image, values below $3 \sigma$ are blanked in the data analysis process. The strongest feature of the $3 \mathrm{~mm}$ continuum result is the main compact source centered at $(\alpha, \delta)=(18: 53: 18,01: 25: 25)$; this structure is in agreement with the $3 \mathrm{~mm}$ continuum result of Shepherd et al. (2004) at higher resolution and corresponds to their MM (millimeter) core. Some additional structure is seen along the north-south axis of the cloud. However, interferometric observations of equatorial sources can produce ghost structures along the north-south axis, due to the strong side-lobes that appear in the synthesized beam at such declinations. In the case of our source, the emission seen south of the MM core appears to be associated with IRAS $18507+0121$ (or the UC H II region with a peak emission centered at $(\alpha, \delta)=(18: 53: 19.5,01: 24: 45))$, a source that is clearly seen in Figure 1.

The polarization observed in our continuum map is within the MM core and the UC $\mathrm{H}$ II region, with the highest polarized flux at the MM core. This source has a peak flux of $273 \mathrm{mJy} \mathrm{beam}^{-1}$, an integrated flux of $290 \mathrm{mJy}$ (calculated over a box of $15^{\prime \prime} \times 15^{\prime \prime}$ centered at the reference position), and a peak polarized flux $\mathrm{P}$ of $83 \mathrm{mJy}^{\mathrm{beam}}{ }^{-1}$; the I and $\mathrm{P}$ peaks approximately coincide. The Stokes I image shows some filamentary structure along the north-south axis between the MM core and the UC H II region, consistent with the general cloud morphology (see Figure1); while the polarized flux is concentrated around the cores. The average P.A. of the polarized continuum map is $-8^{\circ} \pm 5^{\circ}$; suggesting polarization along the north-south axis of the filament. The fractional polarization seems uniform over both cores, with an average value of $0.3 \pm 0.07$. However, interferometric observations that 
do not fully sample the $\mathrm{u}-\mathrm{v}$ plane do not produce reliable fractional polarization results, often overstating the fractional polarization, but not significantly affecting the polarization position angle.

The Shepherd et al. (2004) continuum observations, centered at $90 \mathrm{GHz}$, yielded a total flux of $56 \mathrm{mJy}$, about 4 times less than our value. Our observations have an rms noise level of $6 \mathrm{mJy}^{\text {beam }}{ }^{-1}$ with an uncertainty in the calibration of $25 \%$, while Shepherd et al. (2004) achieved an rms noise level of $3 \mathrm{mJy} \mathrm{beam}^{-1}$ with a calibration uncertainty of $15 \%$. Assuming an error of $3 \sigma$ and taking into account the uncertainty in the calibration, the lower limit for our measured flux is $200 \mathrm{mJy}$. By the same argument, the upper limit for the flux measured by Shepherd et al. (2004) is $75 \mathrm{mJy}$. The remaining ratio of 2.2 between the two measurements is due mainly to the different frequencies. Emission from a blackbody will produce larger values at higher frequencies in the millimeter part of the electromagnetic spectrum; for example, the flux at $115 \mathrm{GHz}$ will be about twice that at $90 \mathrm{GHz}$ for a $50 \mathrm{~K}$ source. The small remaining difference could be explained by missing flux in the higher resolution interferometric observations of Shepherd et al. (2004) due to missing short spacings in $\mathrm{u}-\mathrm{v}$ space. Our compact BIMA D array observations have fairly good shortspacing coverage, with shortest baselines of $\sim 9$ meters, which provides sensitivity up to structures $\sim 60^{\prime \prime}$ in size.

Emission in the continuum at $3 \mathrm{~mm}$ may be contaminated by free-free emission, but it appears not to be the case here. Shepherd et al. (2004) found marginal free-free continuum emission in the $6 \mathrm{~cm}$ band that is estimated to be $0.52 \mathrm{mJy}$ at $3 \mathrm{~mm}$. This free-free emission is unpolarized and negligible when compared with our total flux at $3 \mathrm{~mm}$. However, synchrotron emission, which is strongly linearly polarized, could be present. The polarization of synchrotron radiation in the case of a homogeneous magnetic field can achieve a level of $72 \%$ for a power law emission of $n=0.75$ (Rohlfs \& Wilson 2004). The total level of emission found at $6 \mathrm{~cm}$ was only $0.7 \mathrm{mJy}$, which would yield linearly polarized radiation about 0.5 mJy. However, due to the $\nu^{-n}$ scaling law for synchrotron emission, its contribution to the polarized flux at the $3 \mathrm{~mm}$ band is negligible. Therefore, the contamination in our polarized flux appears to be minimal. This strongly suggests that we are seeing only polarized emission from dust.

\section{2. $\mathrm{CO} J=1 \rightarrow 0$}

The Rathborne et al. (2005) work featured spectra with broad line widths, suggesting evidence for outflow emission towards the MM core; this was later confirmed by Shepherd et al. (2007), who discovered 5 massive outflows. Our CO $J=1 \rightarrow 0$ emission shows similar fea- 
tures for outflow emission; the composite plot in Figure 3 shows the blue and red lobes of the $\mathrm{CO} J=1 \rightarrow 0$ emission. We used, approximately, the same velocity ranges used by Shepherd et al. (2007) to define the outflow lobes (60 to $80 \mathrm{~km} \mathrm{~s}^{-1}$ for the red lobe and 38 to $54 \mathrm{~km} \mathrm{~s}^{-1}$ for the blue lobe). Individual channel maps are shown in Figures 4 and 5 (including the polarization). Considering the larger beam of our BIMA D-array observations, which will smear out the emission, our results are in agreement with the higher resolution observations of Shepherd et al. (2007). Although, we did not resolve all of the individual outflows detected by their work, it appears that the massive outflow in the MM core (outflow A) is dominating the emission in our observations. Our peak CO flux is calculated around the MM source, centered at $(\alpha, \delta)=(18: 53: 17.3,01: 25: 15)$, and taking a value of $\sim 14 \mathrm{Jy}$ beam $^{-1}$. Table 1 presents the average P.A. for all channels associated with polarized CO emission. From Table 1 we see polarized emission at higher velocities (105 to $97 \mathrm{~km} \mathrm{~s}^{-1}$ ) and at more intermediate velocities associated with the MM outflow. The polarization is observed to be quite uniform, with an orientation mostly along the north-south axis of the filament, in all velocity channels shown in Figures 4 and 5. The peaks in polarized flux and polarization coverage is around 64 to $62 \mathrm{~km} \mathrm{~s}^{-1}$, which coincides with the peaks in CO emission. This uniform polarization pattern is similar to the polarization orientation of our $3 \mathrm{~mm}$ continuum results. Taking into consideration that molecular line polarization can be either parallel or orthogonal to the magnetic field, these results reinforce the polarized dust emission results - a magnetic field orthogonal to the main axis of the filament.

\section{Analysis}

\subsection{Core Mass Estimation}

The G34+0.23 MM column density and mass are estimated from dust emission. We follow the derivation made by Mezger (1994). This derivation uses a parametrized representation of the dust absorption cross section per H-atom. This parametrization follows $\tau_{\nu} / N_{\mathrm{H}}=\sigma_{\lambda}^{\mathrm{H}}$ in $\mathrm{cm}^{2} / \mathrm{H}$-atom, where

$$
\sigma_{\lambda}^{\mathrm{H}}=Z / Z_{\odot} b\left(7 \times 10^{-21} \lambda_{\mu m}^{-2}\right) \lambda_{\mu m} \geq 100
$$

$Z / Z_{\odot}=1$ is the relative metalicity, $\lambda_{\mu \mathrm{m}}$ is the wavelength in $\mu \mathrm{m}$, and $b$ is parameter used to introduce the grain dependence on gas density (Mezger 1994). The $b$ parameter usually takes values of $b=1.9$ for $\mathrm{n}_{\mathrm{H}} \leq 10^{6} \mathrm{~cm}^{-3}$ and $b=3.4$ for higher densities. With these

considerations, the expressions for the cloud mass and column density are calculated using the expressions derived by Mooney et al. (1995) 


$$
\begin{aligned}
N_{\mathrm{H}} / \mathrm{cm}^{-2} & =1.93 \times 10^{15} \frac{\left(S_{\nu, \text { int }} / \mathrm{Jy}\right) \lambda_{\mu \mathrm{m}}^{4}}{\left(\theta_{s} / \operatorname{arcsec}\right)^{2}\left(Z / Z_{\odot}\right) b T} \frac{e^{x}-1}{x} \\
M_{\mathrm{H}} / \mathrm{M}_{\odot} & =4.1 \times 10^{-10} \frac{\left(S_{\nu, \text { int }} / \mathrm{Jy}\right) \lambda_{\mu \mathrm{m}}^{4} D_{\mathrm{kpc}}^{2}}{\left(Z / Z_{\odot}\right) b T} \frac{e^{x}-1}{x}
\end{aligned}
$$

where $N_{\mathrm{H}}=N(\mathrm{H})+2 N\left(\mathrm{H}_{2}\right)$ is the total hydrogen column density, $S_{\nu \text {,int }}$ is the integrated flux density from the source, $\theta_{s}=\sqrt{\theta_{s, \min } \times \theta_{s, \max }}$ is the angular source size, $x=\frac{1.44 \times 10^{4}}{\lambda_{\mu m} T}$ is the $\frac{h c}{\lambda k T}$ factor for the Planck function, $D_{\mathrm{kpc}}$ is the distance to the source in kpc, $T$ is the dust temperature, and $b$ is taken to be $b=3.4$ to reflect the massive MM core. Shepherd et al. (2004) estimated $T=50 \mathrm{~K}$ as the most likely dust temperature for the MM core, the value that we use here. Using the flux of $290 \mathrm{mJy}$ estimated from our $3 \mathrm{~mm}$ continuum emission, we obtain a column density of $6 \times 10^{23} \mathrm{~cm}^{-2}$ and a total mass of $520 \mathrm{M}_{\odot}$. Shepherd et al. (2004) obtained a mass of $250 \mathrm{M}_{\odot}$ (their masses varied up to $650 \mathrm{M}_{\odot}$ for different values of dust temperature and emissivity).

\subsection{Field Direction}

The general morphology of the G34.4 massive star forming region is of a filament, where all sources are embedded along the main axis of the cloud (see Figure 1 and maps in Rathborne et al. (2005); Shepherd et al. (2007)). This type of morphology appears to be widespread in the ISM (Faúndez et al. 2004). How this elongated cloud morphology is produced is still an open question; one avenue of research is to understand the dynamical effect of magnetic fields on the gas and dust in these regions. Our dust polarized emission results show a uniform pattern over both the MM core and the UC H II region, with an average P.A. of $-8^{\circ} \pm 5^{\circ}$ aligned with the main axis (north-south) of the filament, between the MM core and the UC H II region (see Figure 2). Polarized emission from dust grains suggests alignment of grains by a magnetic field; the aligned grains will produce polarized emission with P.As. orthogonal to the projection of the field onto the plane of the sky. Therefore, our observations suggest a magnetic field morphology with field lines orthogonal to the main axis of the filament between the MM core and the UC H II region.

A similar interpretation applies for our $\mathrm{CO} J=1 \rightarrow 0$ observations, shown in Figures 4 and 5 in velocity channel maps. Table 1 shows the average values for the P.A. for all channel maps with their corresponding spatial dispersion. A P.A. average of $-2^{\circ} \pm 8^{\circ}$ is calculated for all channels. Figure [6 shows the same plot than Figure 3, but with the polarization map overplotted. The image to the left shows an average of channels from 80 to $60 \mathrm{~km} \mathrm{~s}^{-1}$ for the red lobe, while the image to the right shows an average of channels from 54 to $38 \mathrm{~km} \mathrm{~s}^{-1}$ 
representing the blue lobe. The average P.A. angle in the red lobe is $-3^{\circ} \pm 7^{\circ}$, while in the blue lobe it is $-3^{\circ} \pm 9^{\circ}$, consistent with the average and overall values shown in Table 1. Figure 7 shows panels with spectra from the most intense CO $J=1 \rightarrow 0$ emission points. These two spectra were obtained from averages of $8^{\prime \prime}$ boxes around the points $(\alpha, \delta)=(18: 53: 17.3$, 01:25:15) and (18:53:19.2, 01:24:41) which can be easily spotted in Figure 4 at channel map number $30^{\text {th }}$, or at $V=62 \mathrm{~km} \mathrm{~s}^{-1}$, corresponding to the main emission peaks on the map. Superposed on to each spectrum are fractional polarization and P.A. values which are also consistent with Table 1 and with the previous $\mathrm{CO} J=1 \rightarrow 0$ Figures. Interesting is to see how the fractional polarization values are fairly constant over most of the $\mathrm{CO}$ emission at both spectra, while the P.A. values are well clustered around $0^{\circ}$. The large fractional $\mathrm{CO}(J=1 \rightarrow 0)$ polarization values obtained in our observations can be explained, most likely, by missing flux in the Stokes I emission due to incomplete u-v coverage at the shorter baselines of the BIMA D array configuration.

As with the polarized dust emission results, the polarization line segments seem to be well aligned with the filament axis, showing little spatial dispersion over all relevant channels. Cortes et al. (2005) showed that strong large velocity gradients will produce polarization perpendicular to the magnetic field even if a weak continuum source is present, which seems to be the case in our observations. Therefore, our results for both polarized dust and CO $J=1 \rightarrow 0$ results suggest a magnetic field orthogonal to the main axis of the filament. Flattened filamentary cores with magnetic field lines perpendicular to their main axis have been observed before. The Schleuning (1998) map of $8^{\prime} \times 8^{\prime}$ of the OMC-1 region shows a magnetic field that is not only orthogonal to the main axis of the cloud but also has an hourglass shape. Cortes et al. (2006) made interferometric observations with BIMA towards the NGC2071IR star forming region, finding a magnetic field orthogonal to the main axis of an elongated structure.

It has been also suggested that CO line polarization will, most likely, trace a different environment from dust polarization. Cortes et al. (2005) showed that radiation from the CO $J=1 \rightarrow 0$ transition will present a maximum amount of fractional polarization when emitted from densities $n_{\mathrm{H}_{2}} \sim 100 \mathrm{~cm}^{-3}$ which will, most likely, correspond to cloud envelopes, like the proposed cold screen by Ramesh et al. (1997), or to outflow extended regions. In the case of G34.4, the data support a magnetic field aligned with the main axis of the cloud even at regions dynamically dominated by the outflows. However, polarized line emission tracing outflows aligned with magnetic fields has been observed (Girart et al. 1999; Cortes et al. 2006). This apparent discrepancy might be explained by the difference in spatial resolution between this work and previous findings; while Cortes et al. (2006) interferometric observations achieved a resolution of $4^{\prime \prime}$ from NGC2071IR (at a much closer distance than G34.4), this work is presenting observations at a coarser resolution of $16^{\prime \prime}$. A larger beam may smear 
out the polarization P.As. erasing the outflow signature which will produce a more uniform polarization pattern.

Taking into account that dust emission will trace regions at higher densities $\left(\mathrm{n}_{\mathrm{H}_{2}} \geq 10^{5}\right.$ $\mathrm{cm}^{-3}$ ), which in this case corresponds to the MM core and the UC H II region. Both polarization results suggest a magnetic field perpendicular to the filament at different densities. Interesting would be to have higher resolution observations to map the polarized emission from the outflows in this region.

\section{Summary and Conclusions}

The G34.4+0.23 MM massive star forming core was observed in the $3 \mathrm{~mm}$ band in polarized continuum and in $\mathrm{CO} J=1 \rightarrow 0$ polarized line emission with the BIMA array. The data show a uniform polarization pattern in both emissions. The P.A. obtained from the continuum data has an average value of $\langle\phi\rangle=-8^{\circ} \pm 5^{\circ}$, and from the CO polarization $<\phi>=-2^{\circ} \pm 8^{\circ}$. These results suggest a magnetic field perpendicular to the main axis of the filament, between the MM source and the UC H II region, in G34.4. The morphology suggests a flattened disk with the magnetic field along the minor axis, as predicted by the theory of magnetically supported molecular clouds. From our $3 \mathrm{~mm}$ continuum observations, we estimate a total core mass of $400 M_{\odot}$, in agreement with previous observations. Both line and continuum emission agrees in morphology with previous work by Shepherd et al. (2004, 2007). Finally, additional observations, particularly higher resolution interferometric mapping along the filament (including the most northern source seen in Figure 1), will help to constrain, in greater detail, the morphology of the field and to obtain estimates of its strength in the plane of the sky.

P. C. Cortes acknowledges support from the ALMA-CONICYT fund for development of Chilean Astronomy through grant 31050003. P. C. Cortes would also like to acknowledge the support given by NCSA and the Laboratory for Astronomical Imaging at University of Illinois at Urbana-Champaign during this research. Finally, P. C. Cortes would like to acknowledge the contribution by Patricio Sanhueza in making Figure 1. R. M. Crutcher acknowledges support from NSF grants AST 05-40459 and 06-06822. L. Bronfman acknowledges support from the Chilean Center for Astrophysics FONDAP 15010003.

\section{REFERENCES}

Briggs, D. S., Schwab, F. R., \& Sramek, R. A. 1999, in Astronomical Society of the Pacific 
Conference Series, Vol. 180, Synthesis Imaging in Radio Astronomy II, ed. G. B. Taylor, C. L. Carilli, \& R. A. Perley, 127-+

Bronfman, L., Nyman, L.-A., \& May, J. 1996, A\&AS, 115, 81

Cortes, P., \& Crutcher, R. M. 2006, ApJ, 639, 965

Cortes, P. C., Crutcher, R. M., \& Matthews, B. C. 2006, ApJ, 650, 246

Cortes, P. C., Crutcher, R. M., \& Watson, W. D. 2005, ApJ, 628, 780

Faúndez, S., Bronfman, L., Garay, G., Chini, R., Nyman, L.-A., \& May, J. 2004, A\&A, 426, 97

Girart, J. M., Crutcher, R. M., \& Rao, R. 1999, ApJ, 525, L109

Glenn, J., Walker, C. K., \& Jewell, P. R. 1997, ApJ, 479, 325

Goldreich, P., \& Kylafis, N. D. 1981, ApJ, 243, L75

Greaves, J. S., Holland, W. S., Friberg, P., \& Dent, W. R. F. 1999, ApJ, 512, L139

Lai, S., Crutcher, R. M., Girart, J. M., \& Rao, R. 2002, ApJ, 566, 925

Lai, S., Girart, J. M., \& Crutcher, R. M. 2003, ApJ, 598, 392

Lai, S. P. 2001, PhD thesis, University of Illinois at Urbana - Champaign, Urbana, IL 61801, available at the Astronomy library at the Astronomy building

Lazarian, A. 2007, Journal of Quantitative Spectroscopy and Radiative Transfer, 106, 225

Mezger, P. G. 1994, Ap\&SS, 212, 197

Miralles, M. P., Rodriguez, L. F., \& Scalise, E. 1994, ApJS, 92, 173

Molinari, S., Brand, J., Cesaroni, R., \& Palla, F. 1996, A\&A, 308, 573

Mooney, T., Sievers, A., Mezger, P. G., Solomon, P. M., Kreysa, E., Haslam, C. G. T., \& Lemke, R. 1995, A\&A, 299, 869

Palla, F., Brand, J., Comoretto, G., Felli, M., \& Cesaroni, R. 1991, A\&A, 246, 249

R. J. Sault, N. E. B. Killeen. 1998, Miriad users guide, BIMA

Ramesh, B., Bronfman, L., \& Deguchi, S. 1997, PASJ, 49, 307 
Rathborne, J. M., Jackson, J. M., Chambers, E. T., Simon, R., Shipman, R., \& Frieswijk, W. 2005, ApJ, 630, L181

Rohlfs, K., \& Wilson, T. L. 2004, Tools of radio astronomy (Tools of radio astronomy, 4th rev. and enl. ed., by K. Rohlfs and T.L. Wilson. Berlin: Springer, 2004)

Scalise, E., Rodriguez, L. F., \& Mendoza-Torres, E. 1989, A\&A, 221, 105

Schleuning, D. A. 1998, ApJ, 493, 811

Schutte, A. J., van der Walt, D. J., Gaylard, M. J., \& MacLeod, G. C. 1993, MNRAS, 261, 783

Shepherd, D. S., Nürnberger, D. E. A., \& Bronfman, L. 2004, ApJ, 602, 850

Shepherd, D. S., Povich, M. S., Whitney, B. A., \& Robitaille, T. P. 2007, ApJ, 276, Submitted

Szymczak, M., \& Kus, A. J. 2000, A\&AS, 147, 181 
Table 1. Averages of fractional polarization and position angles, per channel, of CO $(J=1 \rightarrow 0)$ polarized emission from G34.4

\begin{tabular}{|c|c|c|c|}
\hline $\begin{array}{l}\text { Channel } \\
\text { number }\end{array}$ & $\begin{array}{l}\text { Velocity } \\
{\left[\mathrm{km} \mathrm{s}^{-1}\right]}\end{array}$ & $P_{\mathrm{CO}}$ & $\begin{array}{c}\phi_{\mathrm{CO}} \\
{\left[{ }^{\circ}\right]}\end{array}$ \\
\hline 9 & 104.8 & $0.4 \pm 0.1$ & $-0.2 \pm 7.4$ \\
\hline 10 & 102.7 & $0.4 \pm 0.1$ & $5.2 \pm 7.4$ \\
\hline 11 & 100.7 & $0.4 \pm 0.2$ & $3.5 \pm 8.8$ \\
\hline 16 & 90.5 & $0.3 \pm 0.1$ & $6.4 \pm 9.9$ \\
\hline 17 & 88.5 & $0.3 \pm 0.1$ & $19.6 \pm 9.4$ \\
\hline 20 & 82.4 & $0.3 \pm 0.2$ & $-15.1 \pm 10.0$ \\
\hline 24 & 74.3 & $0.3 \pm 0.1$ & $-4.1 \pm 8.8$ \\
\hline 25 & 72.2 & $0.2 \pm 0.08$ & $4.9 \pm 8.1$ \\
\hline 26 & 70.2 & $0.2 \pm 0.05$ & $-1.2 \pm 7.1$ \\
\hline 27 & 68.2 & $0.2 \pm 0.06$ & $-1.9 \pm 6.8$ \\
\hline 28 & 66.2 & $0.2 \pm 0.06$ & $-0.4 \pm 6.0$ \\
\hline 29 & 64.1 & $0.3 \pm 0.07$ & $-2.3 \pm 5.7$ \\
\hline 30 & 62.1 & $0.2 \pm 0.07$ & $-8.87 \pm 6.3$ \\
\hline 31 & 60.1 & $0.2 \pm 0.07$ & $-13.7 \pm 7.4$ \\
\hline 32 & 58.0 & $0.4 \pm 0.2$ & $-12.9 \pm 8.0$ \\
\hline 34 & 54.0 & $0.3 \pm 0.2$ & $-2.4 \pm 9.9$ \\
\hline 35 & 51.9 & $0.2 \pm 0.08$ & $0.6 \pm 7.8$ \\
\hline 36 & 49.9 & $0.2 \pm 0.07$ & $-1.9 \pm 7.5$ \\
\hline 37 & 47.9 & $0.2 \pm 0.09$ & $-8.8 \pm 10.0$ \\
\hline 40 & 41.8 & $0.4 \pm 0.2$ & $-4.9 \pm 8.1$ \\
\hline
\end{tabular}




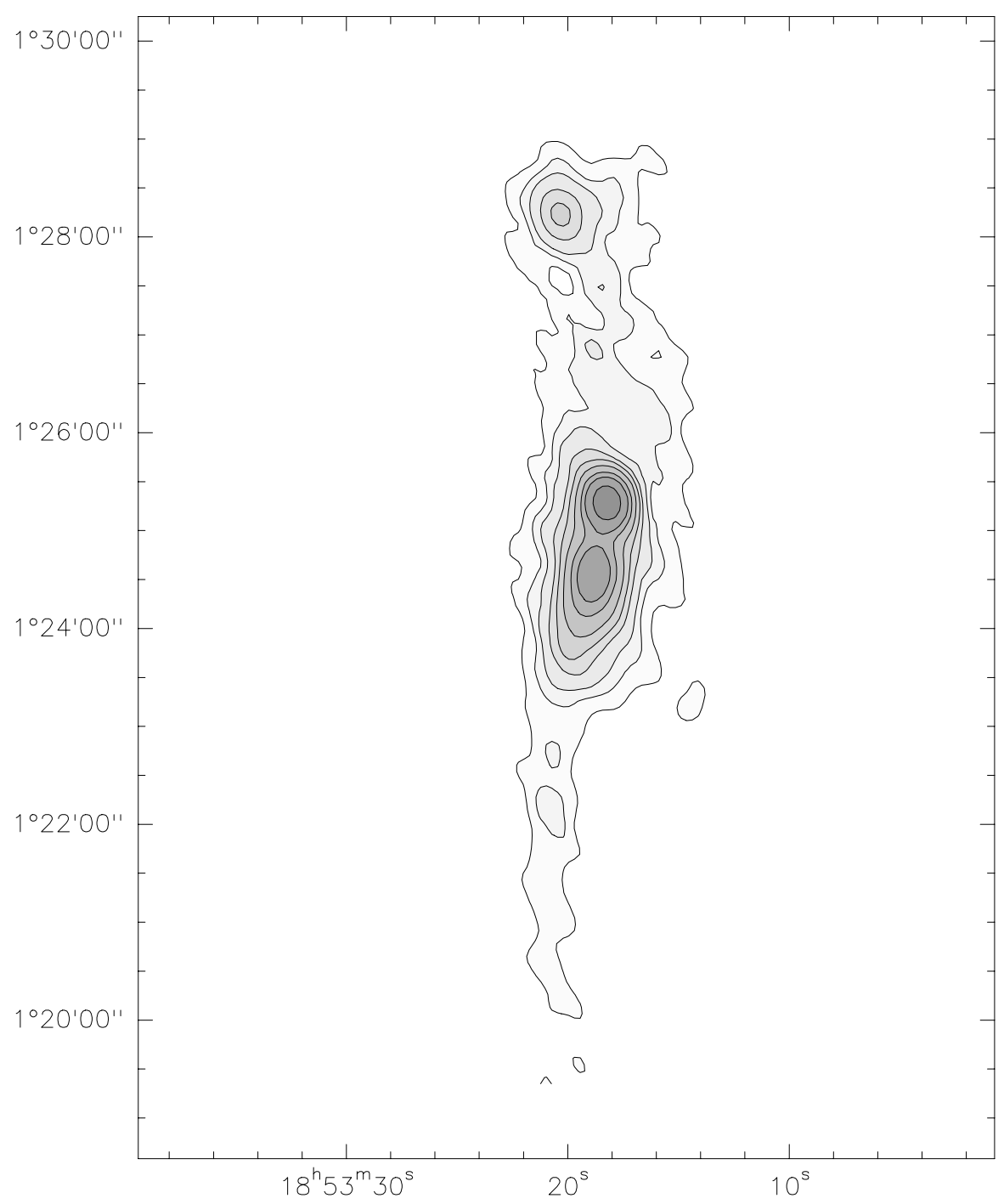

Fig. 1. - The Figure shows the filament which includes the G34.4+0.23 MM source. This Figure was taken from Faúndez et al. (2004), who observed the region at $1.2 \mathrm{~mm}$ with the SEST telescope. We can clearly see the flattened and elongated morphology of the cloud. 


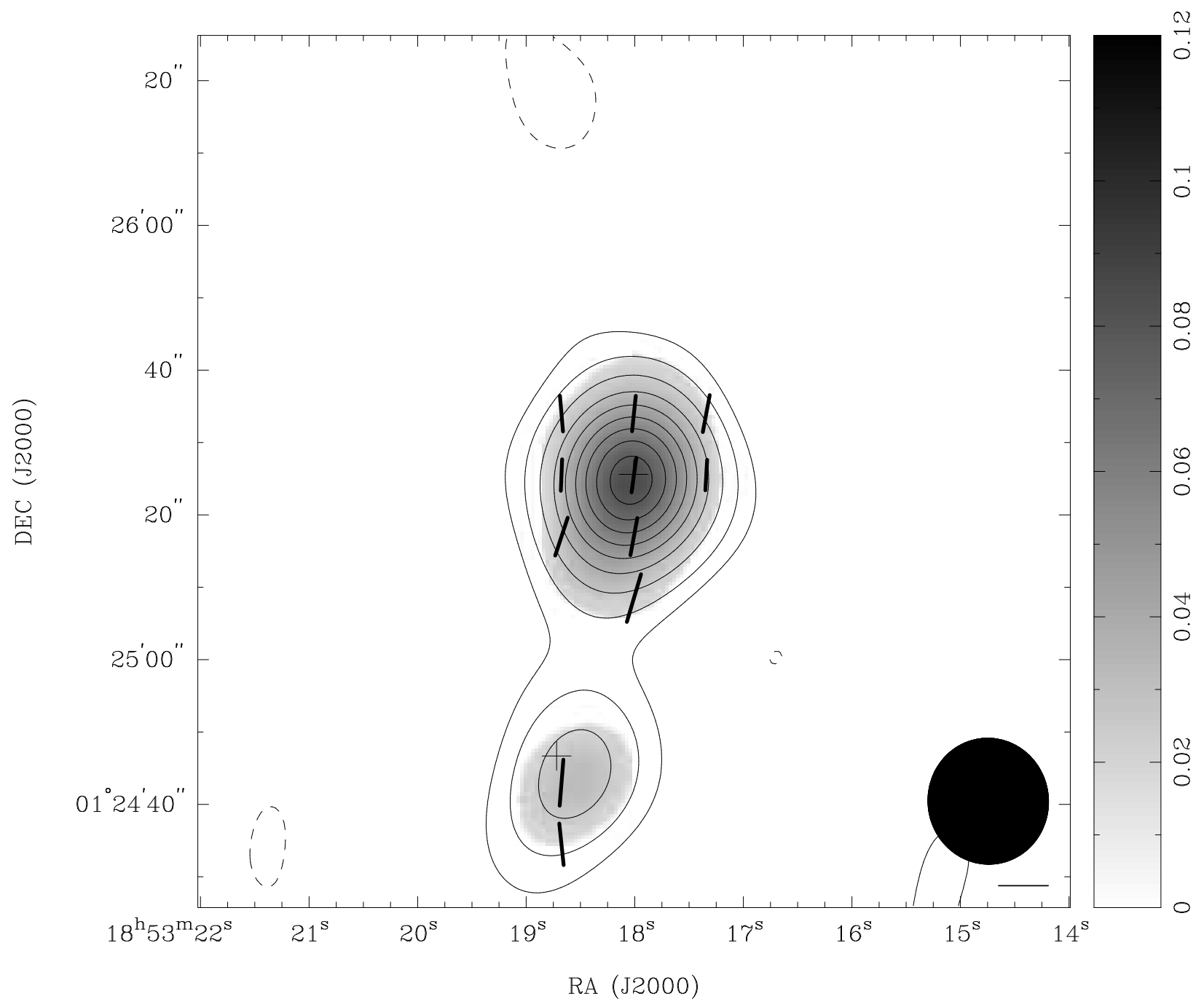

Fig. 2.- The Figure shows the continuum polarization map at $3 \mathrm{~mm}$. This map consists of an intensity only map (or Stokes I) shown by contours at levels of $-3 \sigma, 3 \sigma, 6 \sigma, 10 \sigma, 15 \sigma$, $20 \sigma, 25 \sigma, 30 \sigma, 35 \sigma$, and $40 \sigma$ where $\sigma=6 \mathrm{mJy}_{\text {beam }}{ }^{-1}$. The beam is indicated by the black circle at the bottom rigth corner of the map. The gray scale map indicates the polarized flux $\left(\sqrt{U^{2}+Q^{2}}\right)$, also measured in $\mathrm{Jy}_{\text {beam }}{ }^{-1}$. The line segments show the polarization P.A. and the fractional polarization, which is indicated by the length of the line. The bar at the bottom right, below the beam, shows a fractional polarization scale of 0.4 . The crosses indicate the location of the MM and the UC H II regions. 


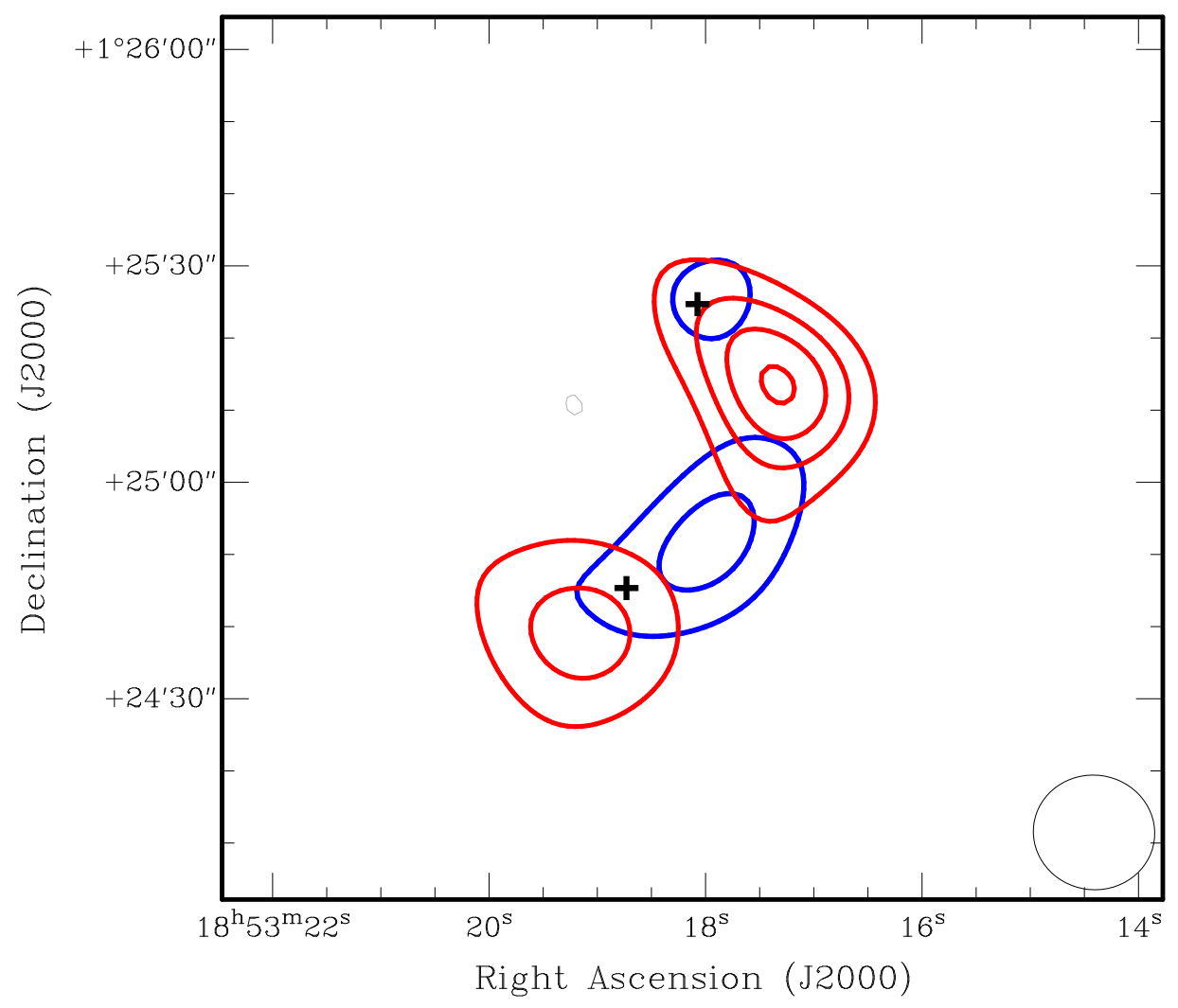

Fig. 3. - A composite plot which superpose the averaged blue shifted lobe (in blue contours) and the red shifted lobe (in red contours), plotted as $-3 \sigma, 3 \sigma, 6 \sigma, 9 \sigma$, and $12 \sigma$ where $\sigma=0.6$ $\mathrm{Jy}_{\text {beam }}{ }^{-1}$. The thick lines represent the positive emission, while the negatives are given by the small gray circle in between both sources. The beam is plotted as a black oval at the bottom right corner. The lobes are calculated using the velocity interval $v=60$ to $v=80$ $\mathrm{km} \mathrm{s}^{-1}$ for the red lobe and $v=38$ to $v=54 \mathrm{~km} \mathrm{~s}^{-1}$ for the blue lobe. As in previous Figures, the crosses represent the MM core and the UC H II region. 


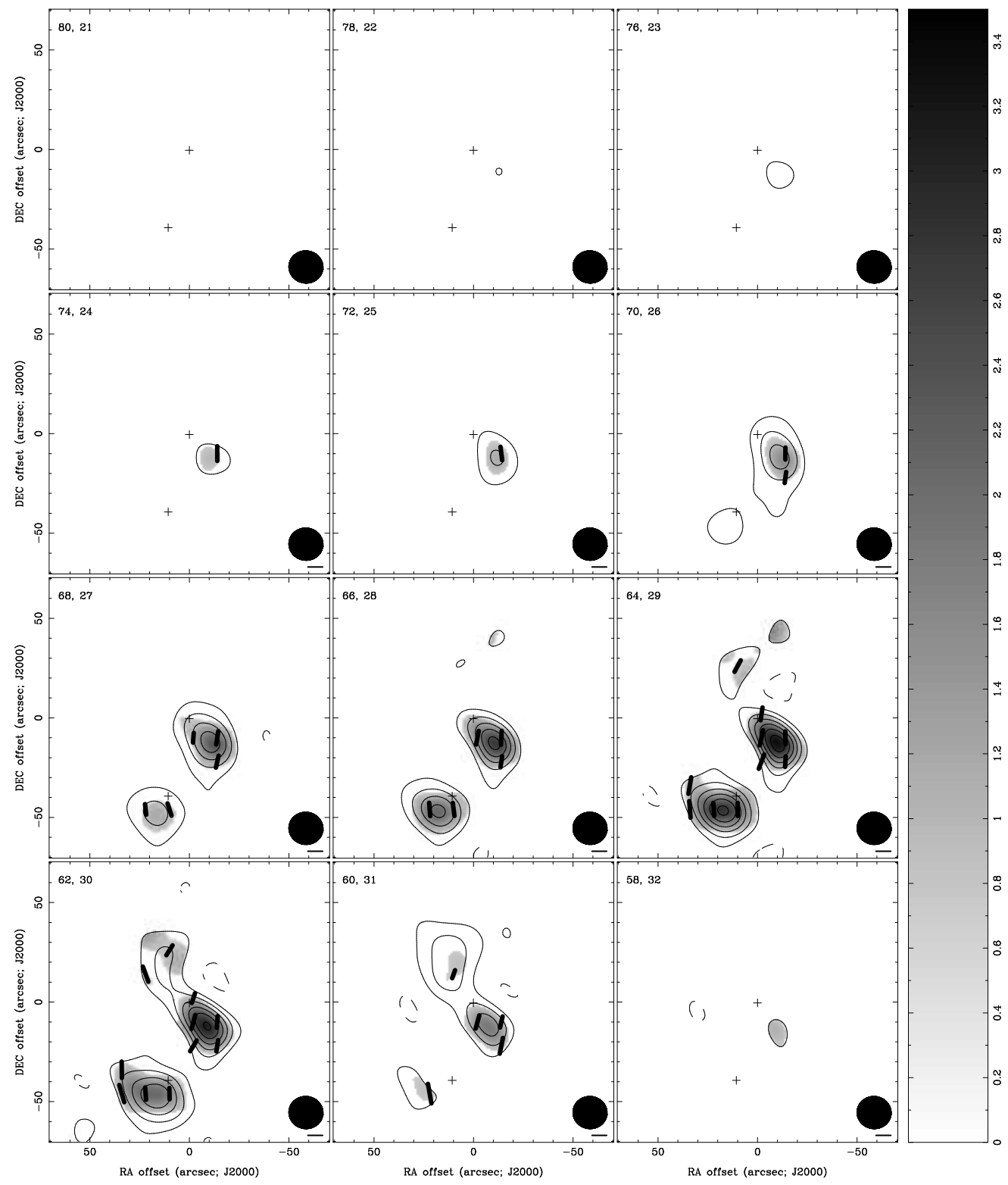

Fig. 4.- Velocity channel maps showing the red-shifted velocity component of the emission. The channel maps have velocity and channel number written at the top left corner of each map; the beam is plotted at the bottom right. The Stokes I emission is plotted as contours of $-3 \sigma, 3 \sigma, 6 \sigma, 9 \sigma, 12 \sigma, 15 \sigma$, and $18 \sigma$ where $\sigma=0.7 \mathrm{Jy} \mathrm{beam}^{-1}$. Polarized flux is shown as gray scale and the line segments represent fractional polarization and P.A. The bar below the beam is the fractional polarization scale of 0.26 and the crosses show the position of the $\mathrm{MM}$ core and of the $\mathrm{UC} \mathrm{H}$ II region. 


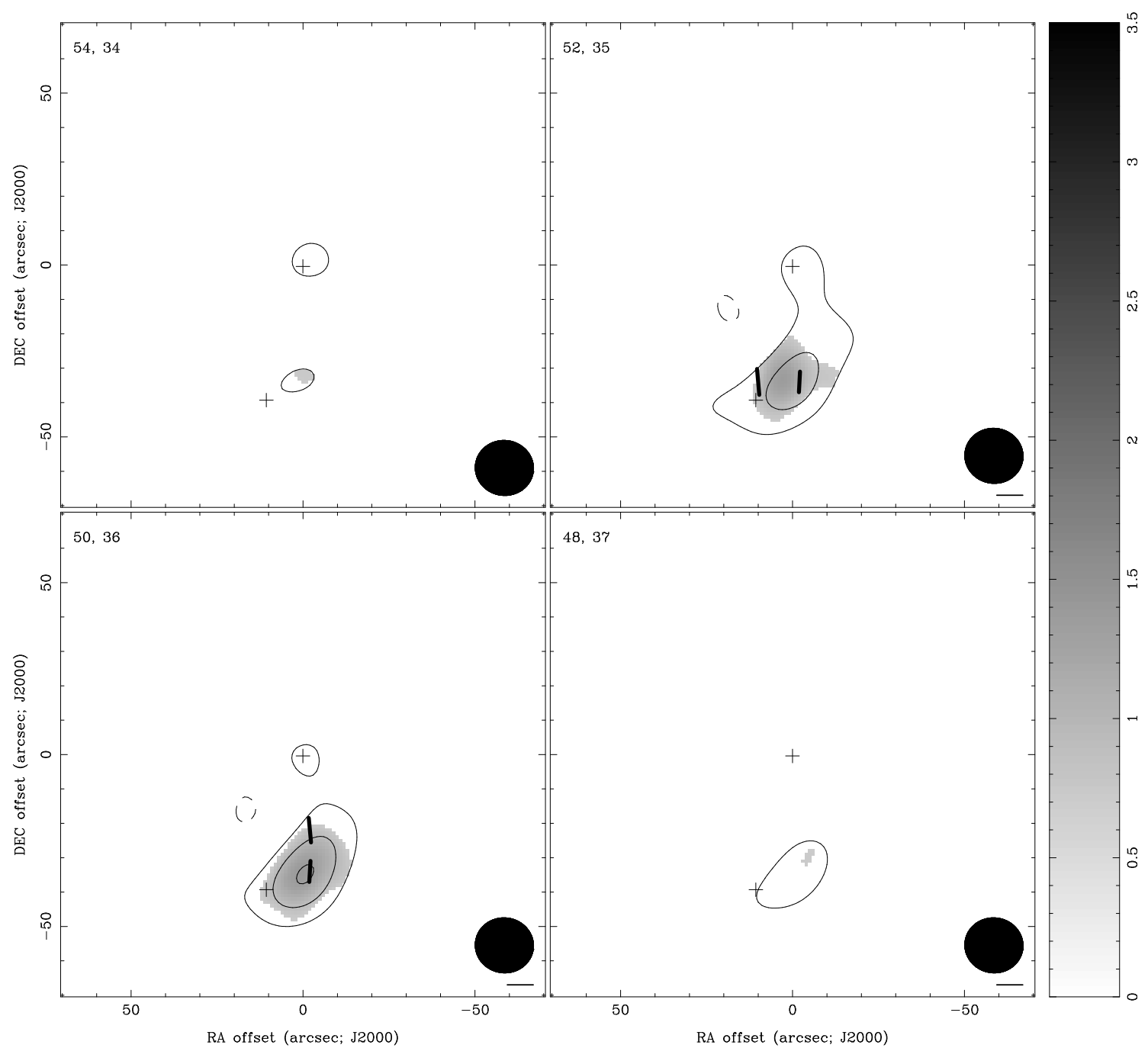

Fig. 5.- Velocity channel maps showing the blue shifted component of the emission. The channel maps have velocity and channel number written at the top left corner of each map; the beam is plotted at the bottom right. As for Figure 4 , the Stokes I emssion is shown as contours of $-3 \sigma, 3 \sigma, 6 \sigma, 9 \sigma, 12 \sigma, 15 \sigma$, and $18 \sigma$ where $\sigma=0.7 \mathrm{Jy} \mathrm{beam}^{-1}$. Polarized flux is shown as gray scale and the line segments represent fractional polarization and P.A. The bar below the beam is the fractional polarization scale of 0.26 and the crosses show the position of the MM core and of the UC H II region. 

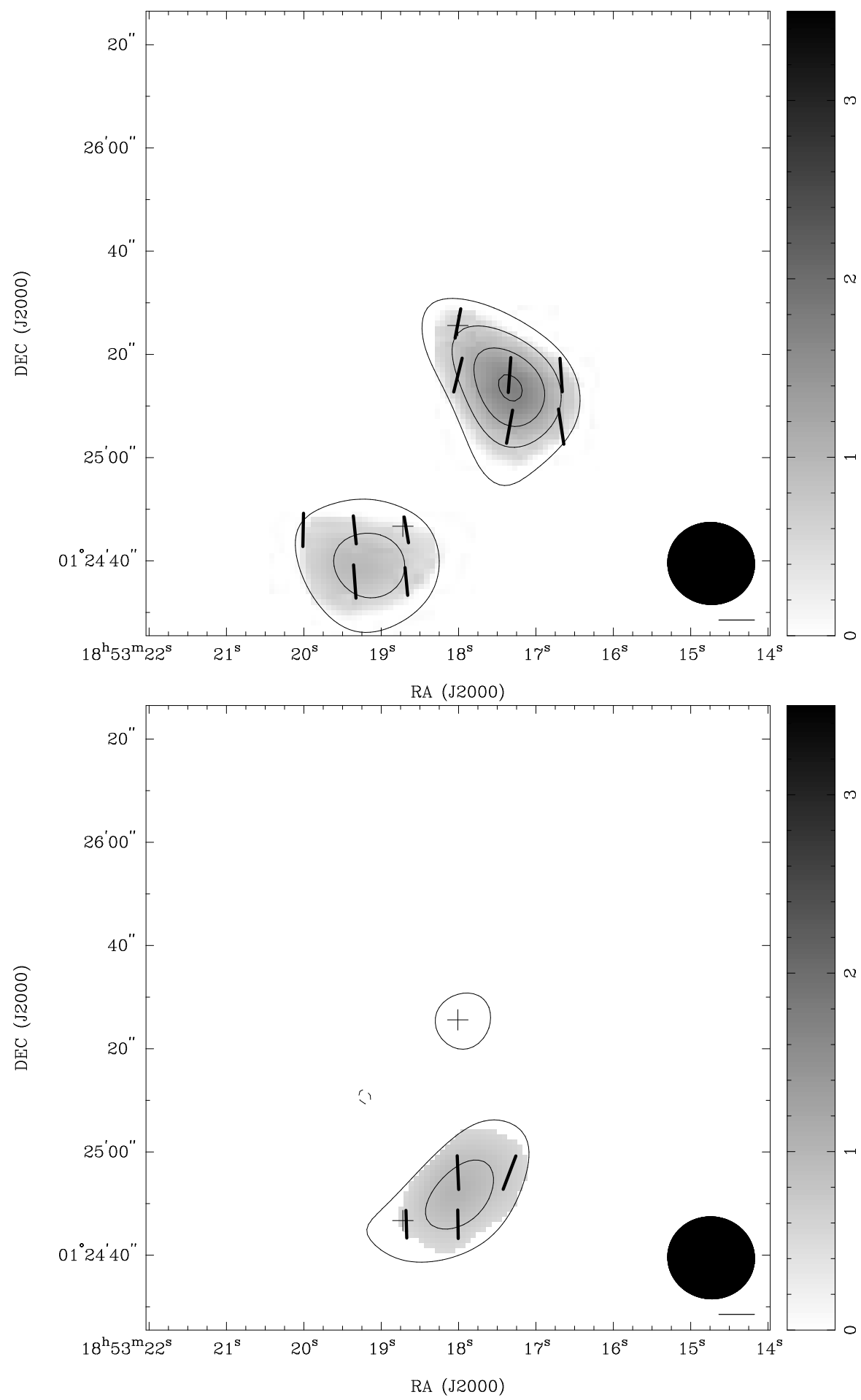

Fig. 6. - The Figure shows two panels with the red shifted (top panel) and blue shifted (bottom panel) emission as shown by Figure 3, but with polarization maps superposed. The Figure shows Stokes I as contours of $-3 \sigma, 3 \sigma, 6 \sigma, 9 \sigma$, and $12 \sigma$, where $\sigma=0.6 \mathrm{Jy} \mathrm{beam}^{-1}$. The small bar below the beam represents the fractional polarization scale in the map, corresponding to 0.22 for the red lobe and 0.25 for the blue lobe. The crosses represent the positions of the MM core and the UC H II region. 

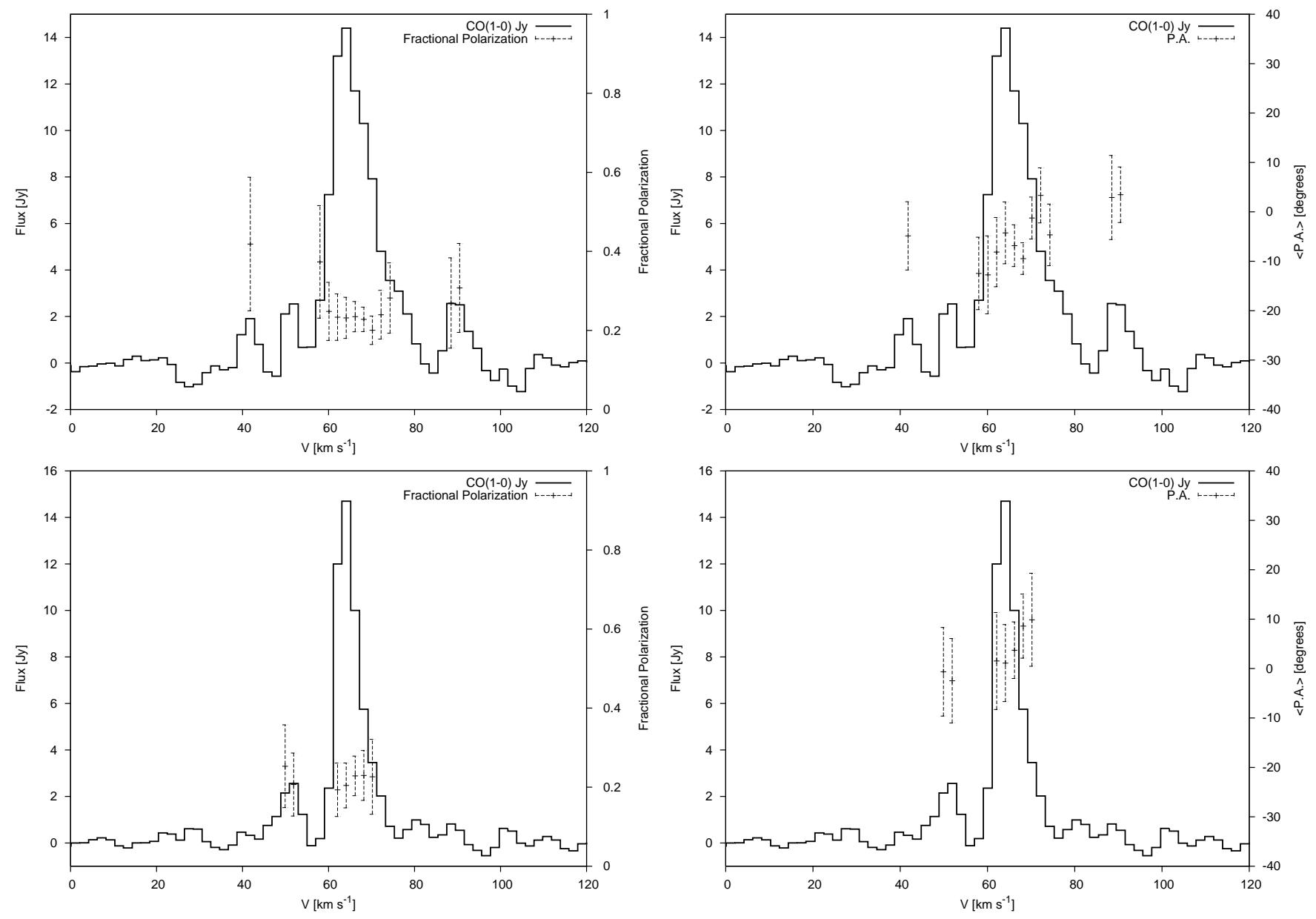

Fig. 7. - Panels show two different spectra from the most intense CO $J=1 \rightarrow 0$ emission points, which are averages taken in $8^{\prime \prime}$ boxes centered at $(\alpha, \delta)=(18: 53: 17.3,01: 25: 15)$ and at (18:53:19.2, 01:24:41). The left panels have superposed the average fractional polarization values, while the right panels have average P.A. values. 